\title{
Motility of small nematodes in wet granular media
}

\author{
G. Juarez, K. Lu, J. Sznitman ${ }^{(a)}$ and P. E. Arratia ${ }^{(b)}$ \\ Department of Mechanical Engineering and Applied Mechanics, University of Pennsylvania, Philadelphia, PA 19104, \\ $U S A$
}

PACS 47.63.Gd - Swimming of microorganisms

PACS 87.85.gj - Movement and locomotion

PACS 45.70.-n - Granular systems

\begin{abstract}
The motility of the worm nematode Caenorhabditis elegans is investigated in shallow, wet granular media as a function of particle size dispersity and area density $(\phi)$. Surprisingly, we find that the nematode's propulsion speed is enhanced by the presence of particles in a fluid and is nearly independent of area density. The undulation speed, often used to differentiate locomotion gaits, is significantly affected by the bulk material properties of wet mono- and polydisperse granular media for $\phi \geq 0.55$. This difference is characterized by a change in the nematode's waveform from swimming to crawling in dense polydisperse media only. This change highlights the organism's adaptability to subtle differences in local structure and response between monodisperse and polydisperse media.
\end{abstract}

Introduction. - Many live organisms evolve within complex fluidic environments such as intestinal fluid, human mucus, and soil [1]3. Such organisms often develop unique methods of locomotion that allow them to exploit the material properties of the media [2,4,5]. For example, in the case of freely swimming spermatozoa in a Newtonian fluid, the flagellum shows a regular sinusoidal beating pattern in both space and time. However, once the organism encounters a complex fluid, like semen, this regular ' beating pattern is replaced by high-amplitude, asymmetric bending of the flagellum [6]. This 'hyper-activated' ' sperm is clearly affected by its fluidic environment, which ' in turn, can affect human fertility [1].

There has been a renewed interest in swimming at low Reynolds numbers [1, 7-11, where live organisms must break time-reversal symmetry [12] in order to achieve net motion. The majority of previous work on locomotion, however, has been restricted to Newtonian liquids, and few investigations are available on the motility of live organisms in complex fluids such as granular media [2, 4, 5, 13]. Recent investigations [4, 14] have indicated that in granular media, an organism needs to tune its swimming amplitude and frequency in order to fluidize the granular

\footnotetext{
(a) Present address: Department of Biomedical Engineering, Technion - Israel Institute of Technology, Haifa 32000, Israel

(b) E-mail: parratia@seas.upenn.edu
}

bed to minimize resistance while keeping the bed rigid enough that it can support applied loads. For millimetersized undulatory swimmers, such as the worm nematode Caenorhabditis elegans ( $C$. elegans), experiments show that the nematode swims more efficiently in a monodisperse particulate system of packing fraction $\phi=0.6$ than in a fluid without particles [13. This observed swimming enhancement was found to be independent of the surrounding particle size.

Despite recent efforts, many important questions remain. For instance, it is known that certain properties of granular media, including packing fraction and particle size dispersity, can significantly affect the response of the bulk material to external perturbations due to the structure of force chains. For example, the response of static quasi-2D piles to an external force behaves more rigid with increasing $\phi$, and for disordered systems the response is best described by elastoplastic models [15 17]. It is yet to be seen how the difference in information propagation between ordered and disordered granular media can affect the motility of live organisms.

Here, we investigate the motility behavior of the nematode $C$. elegans in a buffer solution [18] of viscosity and density similar to water. The solution contains either monodisperse or polydisperse particles of varying area density. Such a fluid can exhibit both solid- and fluid-like behavior depending on packing fraction $\phi$ (referred to here 

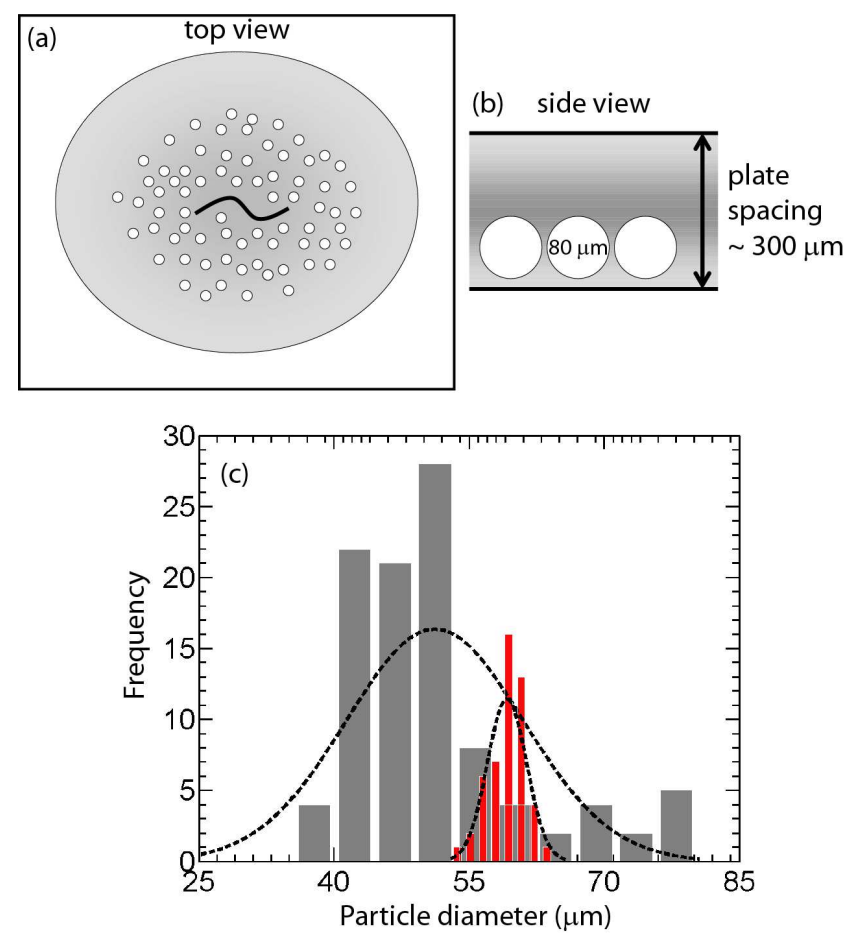

Fig. 1: (Color online) (a) Top view schematic of nematodes swimming in buffer solution $(24 \times 40 \mathrm{~mm})$ and a quasi-2D granular layer $(12 \times 12 \mathrm{~mm})$. (b) Side view schematic of the plate spacing used to confine the nematodes, buffer solution, and granular monolayer. The largest particles used were just under $100 \mu \mathrm{m}$ while the plate spacing was controlled by the volume of buffer solution and was approximately $300 \mu \mathrm{m}$ (c) Plot of the size distribution for monodisperse particles (out of 50 measurements) is shown in red, and for polydisperse particles (out of 100 measurements) is shown in gray.

as particle area density) [19 and is believed to mimic more accurately the soil-like environments where the nematode is originally found. C. elegans is studied extensively and serves as a model organism, and are chosen for this study because they are known to move in different fluidic environments 20 23. They are typically $1 \mathrm{~mm}$ in length, $0.07 \mathrm{~mm}$ in diameter, and are equipped with 95 muscle cells aligning their dorsal and ventral sides 24. Motility is achieved from a 'thrashing' motion against the surrounding medium 25, and typically occurs at a low Reynolds number, $\operatorname{Re} \sim \mathcal{O}\left(10^{-1}\right)$ [26. This makes $C$. elegans an attractive candidate for motility studies.

Experimental methods. - Experiments are performed in a shallow two-dimensional particle bed in order to minimize out-of-plane undulations. The granular monolayer is created by placing the nematode, buffer solution, and particles between two glass cover-slips (170 $\mu \mathrm{m}$ thick), as shown in fig. 1 $(a, b)$. The plate spacing is set by the contact angle between the liquid, the glass cover-slip, and the ambient air as well as the volume of the buffer solution. A constant volume of $400 \mu \mathrm{l}$ of buffer solution is used for each experiment to produce a plate spacing of approximately $300 \mu \mathrm{m}$, which is optically measured. The particles then settle on the bottom plate creating a randomly packed granular layer in which the nematode moves free of confinement effects from the top cover-slip, as shown in fig. 1(b). Note that the total bath size $(24 \times 40 \times 0.3$ $\mathrm{mm})$ is much larger than the granular bed $(12 \times 12 \times 0.08$ $\mathrm{mm}$ ) and the nematode. Monodisperse samples consist of glass beads with diameter $d_{m}=60 \pm 3 \mu \mathrm{m}$ (Mo-Sci Specialty Products) while polydisperse samples consist of glass beads with diameter $d_{p}=52 \pm 10 \mu \mathrm{m}$ (Potters Industries Inc.). A histogram with the measured size distributions for both samples is shown in fig. 1(c).

Two main types of experiments are performed, body tracking methods and particle tracking velocimetry. The motility behavior of $C$. elegans is characterized using body posture tracking methods [27]. The effects of the nematode's motion on the surrounding particles are investigated using particle tracking methods [28. Both C. elegans and particles are imaged using an epi-fluorescent inverted microscope at $5 \times$ magnification (Axio Observer Z1, Carl Zeiss Inc.) with an acquisition rate of 125 frames per second (Fastcam SA1.1, Photron USA Inc.). The C. elegans strain (Genetic Stock Center) is cultured using standard methods 18 .

Nematode kinematics. - The C. elegans' kinematics in wet granular media is analyzed by tracking the nematode body posture in both space and time. Figure2 and supplementary online material shows sample snapshots of the nematode moving in monodisperse (left) and polydisperse (right) wet granular media and the corresponding body postures at a particle area density of approximately 0.55 . An important quantity that can be obtained from body postures is the undulation speed defined as $A f$, where $A$ and $f$ are the nematode's swimming amplitude and frequency, respectively. The beating amplitude $A$ is defined as half of the peak-to-peak distance swept out by the nematode head perpendicular to the swimming direction (fig. 2, top). The beating frequency is the inverse of the period $T$, or the time it takes for the head to complete one sweeping motion. The quantity $A f$ is often used as a relative measure of changes in motility gait 25. Figure 3(a) shows $A f$ as a function of local area density for both monodisperse and polydisperse media. We define the local area density $\phi$ of the granular layer as $\phi=N\left[\bar{d} / 3 L_{n}\right]^{2}$, where $N$ is the total number of spheres, with average diameter $\bar{d}$ of either $62 \mu \mathrm{m}$ for monodisperse media or $52 \mu \mathrm{m}$ for polydisperse media, within an interrogation region locally around the nematode centroid with radius of $\mathcal{R}=3 L_{n} / 4$, where $L_{n}$ is a nematode body length.

Results show that the undulation speed decreases by almost one-half in wet granular media for area density ranging from 0.3 to 0.5 . The undulation speeds are $0.52 \pm 0.05$ $\mathrm{mm} / \mathrm{s}$ and $0.23 \pm 0.05 \mathrm{~mm} / \mathrm{s}$ for buffer solutions $(\phi=0)$ and granular media (mono- and polydisperse media), respectively. This decrease in $A f$ indicates that the nema- 

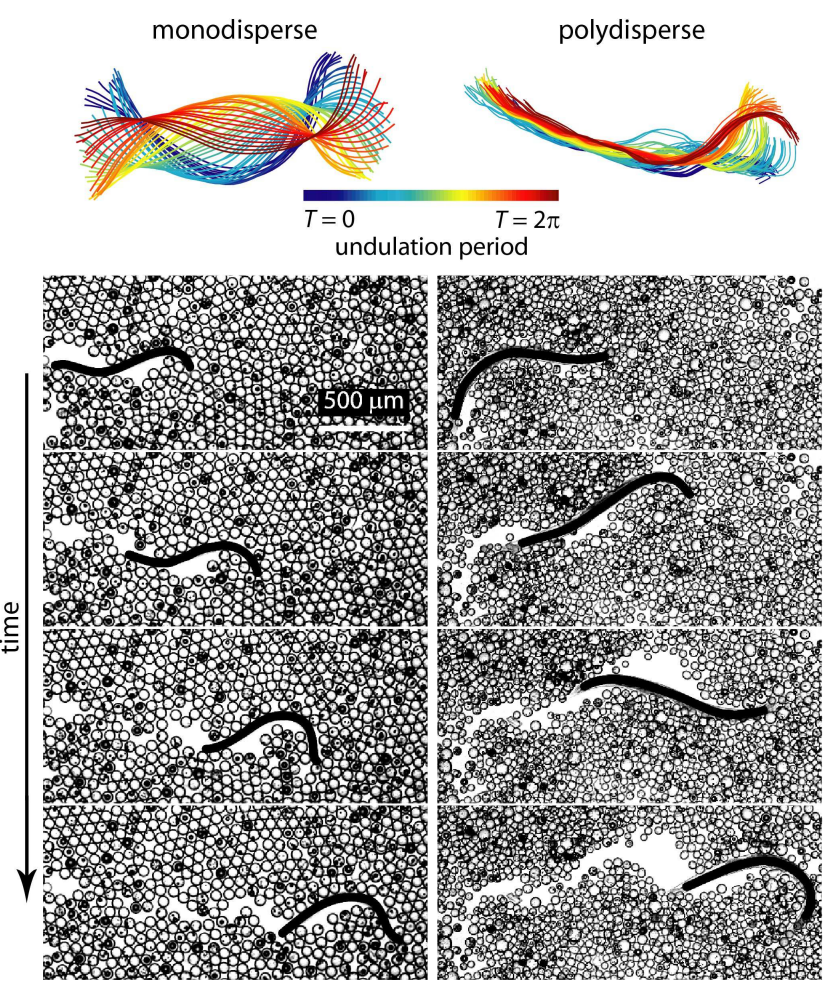

Fig. 2: (Color online) Plots of the nematode skeleton for two environments with particle area densities of 0.55 ; (Left) monodisperse granular media (Right) polydisperse granular media. The undulation period $T$ is color-coded such that blue and red correspond to $T=0$ and $T=2 \pi$, respectively. Below the contours are time-sequenced micrographs of wild-type C. elegans moving through (Left) monodisperse and (Right) polydisperse wet granular media with an area density of 0.55 . Images are taken at equally spaced intervals of $\Delta t=1.12 \mathrm{~s}$ progressing from top to bottom. Note the differences between the bottom two micrographs: (Right) large amount of white space, or void space, free of particles compared to (Left) monodisperse media. See supplementary online material for corresponding movies.

tode moves with slower and/or smaller undulations. For values of $\phi$ larger than 0.55 , the undulation speed is also affected by the media dispersity (fig. 3 $\mathrm{k}$ ). For the polydisperse case, the undulation speed decreases for $\phi \geq 0.55$, whereas $A f$ remains nearly constant for the monodisperse case under similar conditions.

The decrease in undulation speed for area densities larger than 0.55 is accompanied by the nematode's change in motility gait. The change in motility behavior can be seen at the top of fig. 2, which shows the time-evolutions of nematode skeletons over one beating cycle. The contour lines are evenly spaced with intervals of $0.08 \mathrm{~s}$, centered at the nematode's center-of-mass, with the head oriented to the right. The nematode exhibits markedly different waveforms in a buffer solution without particles $(\phi=0)$ than in the same solution containing a high concentration of polydisperse particles $(\phi \geq 0.55)$. For the monodis-

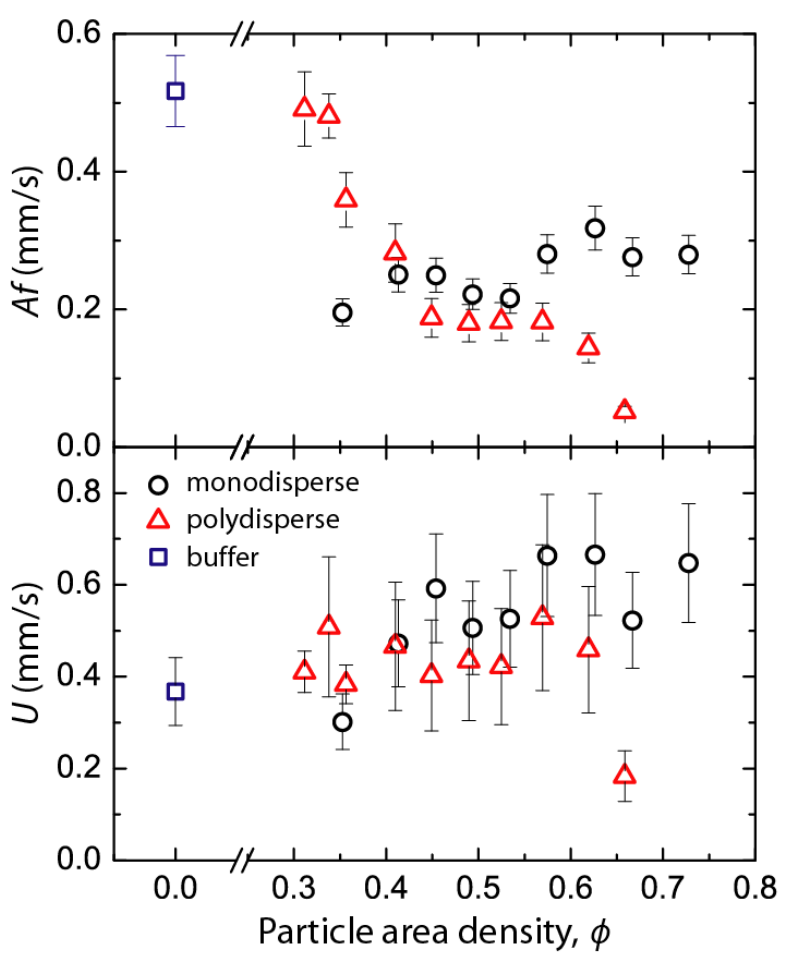

Fig. 3: (Color online) (a) Undulation speed and (b) swimming speed as a function of local area density for C. elegans in wet granular media. The undulation speed decreases drastically in granular media for area densities above 0.3 and remains constant in monodisperse media, but decreases again in polydisperse media beyond $\phi \approx 0.55$. The swimming speed increases slightly in granular media and remains nearly constant with increasing $\phi$.

perse case, the nematode exhibits a waveform identical to the buffer solution $(\phi=0)$ for all values of $\phi$ investigated here. The near-symmetrical waveform seen during swimming (fig. 2. top-left) in a buffer solution or monodisperse media is transformed into an apparent slithering motion as the nematode responds to a dense polydisperse medium (fig. 2, top-right). The peak-to-peak amplitudes for the two cases are approximately $0.36 \mathrm{~mm}$ for $\phi=0$ and 0.175 $\mathrm{mm}$ for $\phi>0.55$. This change in gait was not observed in monodisperse media, and it is indicative of the differences in response and material properties between the two granular media, discussed in more detail in the following section.

Figure 3(b) shows the nematode's propulsion speed $U$ as a function of $\phi$ for monodisperse and polydisperse media. Results show that $U$ increases in wet granular media compared to swimming in a buffer solution, and remains relatively constant, even as $\phi$ increases. The average nematode propulsion speed in buffer solution is $0.36 \pm 0.01$ $\mathrm{mm} / \mathrm{s}$, whereas the propulsion speed in wet granular media is $0.48 \pm 0.12 \mathrm{~mm} / \mathrm{s}$, varying slightly with local area density. Overall, we find that even though the undulation 


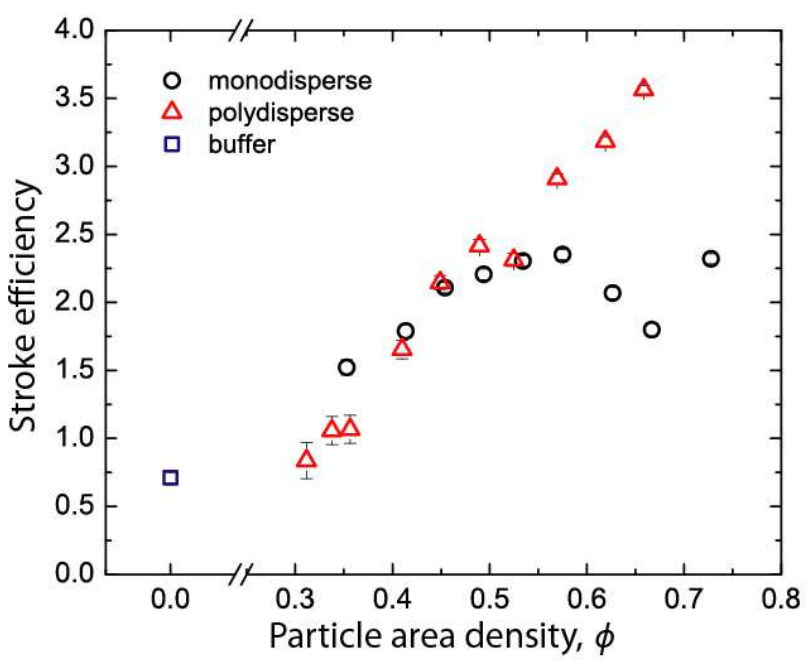

Fig. 4: (Color online) Stroke efficiency, the ratio of propulsion speed $U$ to undulation speed $A f$, as a function of local particle area density for $C$. elegans in wet granular media. The Stroke efficiency increases with area density up to 0.55 and then remains constant at a value of approximately 2.25 for monodisperse media. The Stroke efficiency increases monotonically for polydisperse media.

speed decreased in dense wet granular media, this did not hinder the C. elegans ability to move. This observation can be further quantified by computing the ratio of propulsion speed $U$ to undulation speed $A f$ as a function of $\phi$, referred to here as the Stroke efficiency $\nu=U / A f$, shown in fig. 4. Values of $\nu>1$ imply that there is greater forward swimming speed than transverse undulation speed. We find that $\nu \approx 0.69$ for swimming in a fluid without particles and $\nu \approx 2.08$ for swimming in wet granular media. The Stroke efficiency increases with increasing $\phi$ implying that the nematode moves more efficiently by generating more forward motion with less lateral motion. Obviously, the efficiency cannot be unbounded and is expected to plateau at some value of $\phi$ until reaching the jamming limit. Monodisperse media appears to plateau to a value of $\nu \approx 2.25$ for area densities greater than 0.55 . We note that the Strouhal number, which is the inverse of $\nu$, is typically used to characterize vortex shedding in oscillating flow mechanisms. It was also recently used to suggest that $C$. elegans move more efficiently in granular media [13.

Nematode propulsion in granular media. - It is known that $C$. elegans have distinct motility gaits, such as swimming or crawling, depending on whether the environment is a liquid buffer solution or a solid agar gel, respectively [25]. That is, the nematode is sensitive to the material properties of its surrounding fluidic environment. Here, for $\phi<0.3$, particles are sparse (dilute) and the nematode behaves essentially as if it swimming in a pure buffer solution with no particles. In addition, figs. 3 and 4

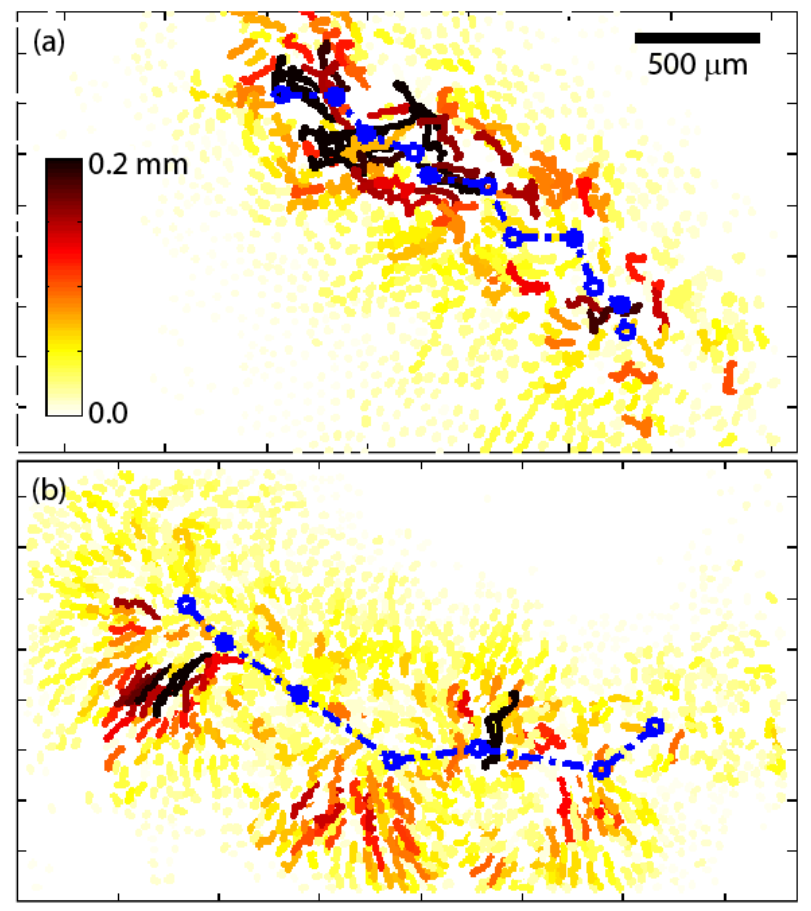

Fig. 5: (Color online) Particle pathlines induced by the nematode in (a) monodisperse and (b) polydisperse media for $\phi \approx 0.55$. The corresponding nematode undulation frequencies are $f_{m} \approx 1.5 \mathrm{~Hz}$ and $f_{p} \approx 0.5 \mathrm{~Hz}$. Particles are tracked for $t=3.1 \mathrm{~s}$. Nematode trajectories are depicted by the blue line with circles representing the nematode center of mass. The length of particle trajectories is color coded such that white and red correspond to short and long trajectories, respectively. The difference in rearrangement to nematode undulations is evident. Monodisperse particle trajectories are localized around the main nematode trajectory, whereas polydisperse particle trajectories extend outward normal to the main nematode trajectory. See supplementary online material for corresponding movies.

show that for area densities in the range of $0.3<\phi<0.55$, the nematode motility behavior is similar for both monoand polydisperse media which indicates that both media have similar material properties. This is due to the lack of large scale structure formation in both media for this semi-dilute density range. However, for $\phi>0.55$, there is an obvious difference in motility behavior that is specific to mono- or polydisperse media as noted by the wave form (fig. 2, top) and undulation speed (fig. 3). This suggests that in this dense regime $(\phi>0.55)$ the bulk material properties of polydisperse media are similar to an elastic material [16,29]30] such as an agar gel, while monodisperse media behave similar to a viscous fluid [31.

In order to gain further insight into the difference in response of mono- and polydisperse media to the nematodes undulations for $\phi>0.55$, we tracked an average of 2,000 particles along the nematodes path. Figure 5 shows particle pathlines for monodisperse (fig. $[5 \mathrm{k}$ ) and polydisperse 

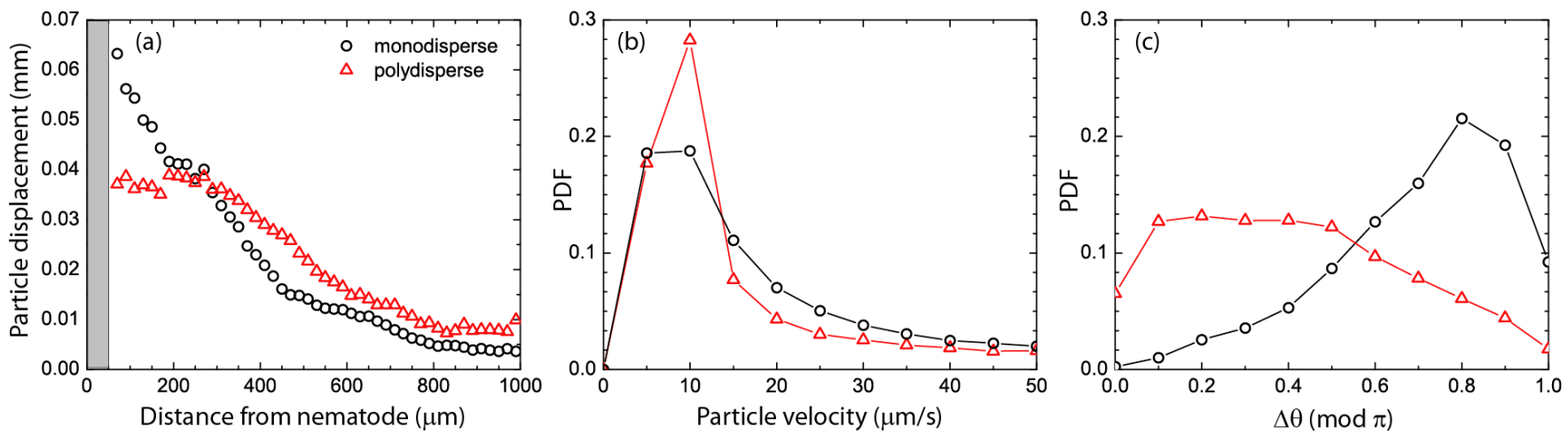

Fig. 6: (Color online) (a) Average overall particle displacement as a function of the radial distance away from the nematode body; the gray region corresponds to the body thickness. Particle displacement is largest near the nematode body and decays rapidly for monodisperse media compared to the polydisperse media. (b) PDF of particle velocities localized around the nematode trajectory. While particle trajectories are longer for monodisperse media (see fig. 5), the probability density function (PDF) of the particle velocities are similar for both monodisperse and polydisperse media. (c) PDF of $\Delta \theta(\bmod \pi)$, the angle between the nematode trajectory and surrounding instantaneous particle velocities. The difference in rearrangement between the two media is evident; the majority of monodisperse particles move nearly anti-parallel to the nematode main trajectory while polydisperse particles move perpendicular to the nematode main trajectory. Note that the lines are added to guide the eye.

(fig. 55) media as a nematode moves through a $3.1 \mathrm{~mm}$ by $1.8 \mathrm{~mm}$ region for $\phi \approx 0.55$. For the monodisperse case, most of the long particle trajectories are localized around and are tangential to the nematode's body. By contrast, for the polydisperse case, particle displacements extend further away from and are mostly normal to the nematode's body. The average displacement of particles nearest the nematode is $\Delta \bar{x}_{m} \approx 60 \mu \mathrm{m}$ and $\Delta \bar{x}_{p} \approx 40 \mu \mathrm{m}$ for monodisperse and polydisperse media, respectively. The average particle displacement decreases rapidly with radial distance from the nematode's body (fig. 6a) ; this decay is expected for viscous fluids. The average particle displacement decays to one-half of its magnitude at a distance of $0.3 L_{n}$ for monodisperse media compared to a distance of $0.55 L_{n}$ for polydisperse media, where $L_{n}=1 \mathrm{~mm}$. As expected, the distributions of particle velocities for both media are similar, which indicates similar energy input from nematode undulations (fig. 6b).

In general, we find that there is a noticeable difference in particle rearrangement behavior between mono- and polydisperse media due to nematode undulations at comparable $\phi$. We characterize this difference by computing the angle between the nematode trajectory and the instantaneous velocity of the surrounding particles, $\Delta \theta(\bmod \pi)$, shown in fig. [6(c). Here, the values of $\Delta \theta(\bmod \pi)=0^{\circ}$ and $\Delta \theta(\bmod \pi)=180^{\circ}$ correspond to the same and opposite direction of swimming, respectively. The data shows that for the monodisperse medium, there is a sharp peak at 0.8 or $\Delta \theta \approx 150^{\circ}$ which indicates that the particles move along the nematode's main trajectory. By contrast, for the polydisperse medium, there is a broad distribution of particle directions mostly moving normal to the nematode's main trajectory. The sample snapshots of the nematode moving in dense granular media (fig. 2 and sup- plementary online movies) illustrates the difference in rearrangement behavior. For the monodisperse case, we observe that the local area density at the tail is only slightly less than at the head (fig. 2, bottom-left) indicating that particles are able to easily rearrange during the perturbation imposed by the moving nematode. This behavior is similar to what is observed for an intruder dragged at constant force through an amorphous monolayer of vibrated grains [32]. For the polydisperse case, however, the overall motion of both small and large particles is mostly laterally from the trajectory of a nematode (fig. 2, bottom-right) and particles do not readily fill the voids, leaving a dilute path behind the nematode. This observation provides further evidence that polydisperse media behaves elastoplastically, by retaining deformations due to nematode undulations.

Overall, we find that $C$. elegans' are able to adapt and utilize the surrounding granular media to convert lateral undulations into net forward motion. This conversion leads to an increase in propulsion speed, which the nematode maintains even with increasing particle area density and degree of size dispersity. The observed enhanced propulsion may be explained by closely inspecting the nematode skeleton during swimming in buffer solutions, (fig. 2, top-left), and recognizing that the motion over one beating cycle is mostly in the lateral direction. As $\phi$ increases, the surrounding particles act as supporting structures that convert undulatory motion into net forward motion. Here, the effective propulsion depends on the difference between the applied load by the nematode undulations and the rigidity of the granular media 4, 14. The rigidity, or ability to withstand deformation, depends on the local particle area density and becomes more solidlike at larger densities, until reaching the jamming limit 
$\phi_{J} \approx 0.84$ [33. This rigidity also depends on the size dispersity, which affects the amount of disorder of the granular media [17,34]. Polydisperse samples are more disordered and therefore present a more "rigid" quasi-static fluidic environment, whereas monodisperse samples are less disordered and are more easily sheared, therefore appear more fluid-like to the C. elegan 35 .

Finally, an estimate of the propulsive force $\left(F_{P}\right)$ can be obtained by computing the work done on the surrounding particles from nematode undulations, $W=F_{P} A-F_{D} x$, where $A$ is the nematode beating amplitude, $F_{D}$ is Stokes' drag, and $x$ is the average displacement of a group of particles. The kinetic energy of the particles is computed from particle tracking data. The propulsive force is estimated by $F_{P}=\pi \rho d^{3}\left[N v_{g}^{2} / 12 A\right]+3 \pi \eta d\left[N v_{g} x / A\right]$. Here $\rho$ is the particle density, $d$ is the particle diameter, $\eta$ is the buffer dynamic viscosity, $N$ is the average number of particles moving, and $v_{g}$ is the average group velocity of the particles per undulation. This approximation reveals that the force of the $C$. elegans on the surrounding media is $F_{P} \sim \mathcal{O}\left(10^{-10}\right)$ Newtons. This value is similar to the force generated by a slender-body swimming in a viscous fluid [26 and is only slightly less than values measured for crawling $C$. elegans on substrates using force sensing pillars [36].

Conclusions. - In conclusion, we have shown that live organisms can exploit the material properties of the surrounding complex media to achieve efficient propulsion. Surprisingly, the addition of particles to a buffer solution does not hinder the $C$. elegans ability to move. In fact, the nematode's propulsion speed is enhanced by the presence of particles and is not dependent on particle area density. The size dispersity on the other hand, does affect the locomotion gait of the nematode at high area densities.

The adaptive motility behavior of $C$. elegans in wet granular media may explain the consistent performance seen not only in nematodes [20, but also in flagellated bacteria and human spermatozoa. Similar mechanisms have been suggested in the enhanced movement of Leptospira 37. and other flagellated bacteria in non-Newtonian fluids. Hence, it is possible that other forms of microscopic propulsion are also tuned to biological fluids which possess anisotropies. In addition, we are able to study the response and rearrangement of the granular media and its dependence on packing fraction and particle size dispersity free of external forces, such as gravity. This suggests an interesting method to probe more complex anisotropic media that could depend on shape or particle interactions.

$$
* * *
$$

We thank X. Shen, D. J. Durian, T. Shinbrot, P. B. Umbanhowar, and J. P. Gollub for providing helpful comments. This work was partially funded by NSF CAREER award CBET-0954084.

\section{REFERENCES}

[1] Fauci L. J. and Dillon R., Annu. Rev. Fluid Mech., 38 (2006) 371 .

[2] Maladen R. D. and et Al., Science, 325 (2009) 314.

[3] Vogel S., Life in moving fluids 2nd Edition (Princeton University Press, Princeton, NJ) 1994.

[4] Shimada T. and et Al., Phys. Rev. E, 80 (2009) 020301.

[5] Mazouchova N. and et AL., Bio. Lett. , 6 (2010) 398.

[6] Ho H. and Suarez S., Reprod. , 122 (2001) 519.

[7] Teran J., Fauci L. and Shelley M., Phys. Rev. Lett. , $104(2010) 4$.

[8] Troullloud R. and et Al., Phys. Rev. Lett. , 101 (2008) 4.

[9] Dreyfus R. and et AL., Nature, 437 (2005) 862.

[10] Polin M. and Et AL., Science, 325 (2009) 487.

[11] Cohen N. and Boyle J. H., Contemporary Physics , 51 (2010) 103.

[12] Purcell E. M., Am. J. Phys. , 45 (1977) 3.

[13] Jung S., Phys. Fluid, 22 (2010) 031903.

[14] Li C. and et Al., Proc. Natl. Acad. Sci. U.S.A. , 106 (2009) 3029.

[15] Luding S., Nature , 435 (2005) 159.

[16] Geng J. and et Al., Phys. Rev. Lett. , 87 (2001) 035506.

[17] Muthuswamy M. and Tordesillas A., J. Stat. Mech. Theory and Exp. , (2006) P09003.

[18] Brenner S., Genetics , 77 (1974) 71.

[19] Larson R. G., The Structure and Rheology of Complex Fluids (Oxford University Press, New York) 1999.

[20] Korta J. and et AL., J. Exp. Biol. , 210 (2007) 2383.

[21] Sznitman J. and Et AL., J. Exp. Mech. , (2010) published on line.

[22] Berri S., Boyle J. H., Tassieri M., Hope I. A. and Cohen N., HFSP J. , 3 (2009) 186.

[23] Park S. and et Al., PLOS One, 3 (2008) e2550.

[24] White J. G., Southgate E., Thomson J. N. and ET Al., Phil. Trans. R. Soc. Lond. B Biol. Sci. , 314 (1986) 1.

[25] Pierce-Shimomura J. T. and et Al., Proc. Natl. Acad. Sci. USA, 105 (2008) 20982.

[26] Sznitman J. and ET AL., , (2010) submitted.

[27] Sznitman J. and et AL., Biophys. J. , 98 (2010) 617.

[28] Crocker J. C. and Grier D. G., J. Colloid Interface Sci. , 179 (1996) 298.

[29] Bouchaud J.-P., Claudin P., Levine D. and Отto M., Eur. Phys. J. E , 4 (2001) 451.

[30] Goldenberg C. and Goldhirsch I., Phys. Rev. Lett. , 89 (2002) 084302.

[31] Jaeger H. M., Nagel S. R. and Behringer R. P., Rev. Mod. Phys. , 68 (1996) 1259.

[32] Candelier R. and Dauchot O., Phys. Rev. Lett. , 103 (2009) 128001.

[33] Donev A. and et AL., J. Appl. Phys., 95 (2004) 989.

[34] Geng J., Reydellet G., Clément E. and Behringer R. P., Physica D , 182 (2003) 274.

[35] Bardenhagen S. G. and Brackbill J. U., Phys. Rev. E, 62 (2000) 3882 .

[36] Doll J. C. and ET Al., Lab Chip, 9 (2009) 1449.

[37] Kaiser G. E. and Doetsch R. N., Nature, 255 (1975) 656. 